\title{
Evaluating the evolving evidence: The challenges of molecular-targeted therapy in management of gastric
} cancer

\author{
Yasar Albushra Abdul Rahiem Ahmed ${ }^{1}$, Mohamed Ali $^{2}$ \\ ${ }^{1}$ Department of Medicine, Wad Medani Teaching Hospital, Wad Medani, Sudan \\ ${ }^{2}$ Advanced Oncology Research, Department of Medical Oncology, National Cancer Institute, Khartoum, Sudan \\ Email: $\underline{\text { medicine2u@yahoo.co.uk }}$
}

Received 25 November 2013; revised 29 December 2013; accepted 8 January 2014

Copyright (C) 2014 Yasar Albushra Abdul Rahiem Ahmed, Mohamed Ali. This is an open access article distributed under the Creative Commons Attribution License, which permits unrestricted use, distribution, and reproduction in any medium, provided the original work is properly cited. In accordance of the Creative Commons Attribution License all Copyrights (C) 2014 are reserved for SCIRP and the owner of the intellectual property Yasar Albushra Abdul Rahiem Ahmed, Mohamed Ali. All Copyright (C) 2014 are guarded by law and by SCIRP as a guardian.

\section{ABSTRACT}

Over the past decade, a multitude of molecular targeted agents have been explored in the treatment of advanced metastatic gastric. Recent advances in molecular signaling pathways that are dysregulated in gastric cancer lead to the development of new targeted therapies for the treatment of advanced and metastatic gastric cancer. The addition of trastuzumab to first-line chemotherapy is now a standard of care for the treatment of Human epidermal growth factor receptor (HER2-) positive advanced or metastatic disease, and other HER2-targeted therapies are in late-stage clinical development. Findings from recent major clinical trials provide important insight into the future of metastatic gastric cancer management, which may include the use of anti-angiogenesis, Mesenchymal epithelial transition factor (MET) and Hedgehog Pathways Inhibitortherapy across multiple treatment lines, in the salvage setting, and as part of novel regimens in combination with other targeted agents.

\section{KEYWORDS}

Gastric Cancer; Targeted Therapy; Trastuzumab; HER2-

\section{INTRODUCTION}

Gastric cancer is a major health problem around the world [1]. In Asian countries where the disease is highly prevalent, screening facilitates early detection, but in Middle East and Western countries, the disease is typi- cally diagnosed at an advanced, incurable stage [1,2]. Chemotherapy remains the treatment of choice for patients who have unresectable locally advanced, locally recurrent, or metastatic gastric cancer and an acceptable performance status [1]. In the United States, 2-drug regimens are generally preferred, although 3-drug regimens can be used to treat medically fit patients who have a good performance status and access to frequent evaluation for toxicity [1]. Despite the availability of a variety of different active regimens, the fatality-to-case ratio remains high [3]. Five-year survival is less than $10 \%$, and median survival time is less than 1 year [4].

Consistent with trends occurring in the treatment of other solid tumors, researchers are evaluating the safety and effectiveness of different targeted therapies for the treatment of advanced and metastatic gastric cancer. Targeted therapy is an attractive approach in this setting due to the limited efficacy of chemotherapy, its proven benefit in other cancers, and the lack of overlapping toxicity between targeted agents and chemotherapy [1] Currently, one targeted therapy, trastuzumab, is approved for the treatment of advanced human epidermal growth factor receptor (HER)-positive gastric cancer, but others are in late-stage clinical development [5].

This activity focuses on the following molecular signaling pathways that are dysregulated in gastric cancer and the corresponding agents that are in late-stage development (Figure 1) [2]:

- Human epidermal growth factor receptor (HER)

- Vascular endothelial growth factor (VEGF)

- Mesenchymal epithelial transition factor (MET)

- Hedgehog (Hh) 


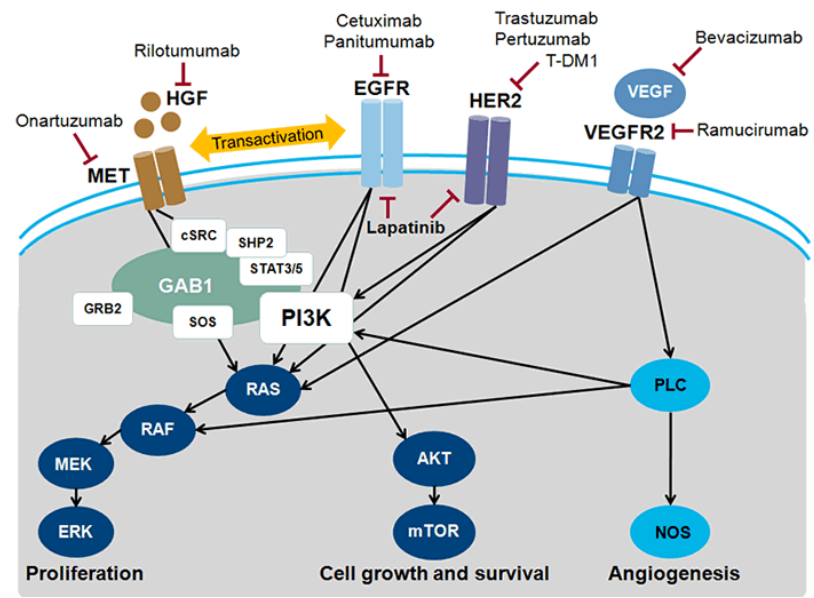

Figure 1. Molecular targets and inhibitory agents in advanced gastric cancer. $\mathrm{AKT}=$ protein kinase $\mathrm{B}$; $\mathrm{cSRC}=$ tyrosine protein; EGFR = epidermal growth factor; receptor; ERK = extracellular signal-regulated kinase; GAB1 = GRB2-associatedbinding protein 1; GRB2 = growth factor receptor-bound protein 2; HER = human epidermal growth factor receptor; HGF = hepatocyte growth factor; MEK = MAP kinase kinase; $\mathrm{MET}=$ MET receptor; $\mathrm{mTOR}=$ mammalian target of rapamyin; NOS = nitric oxide synthase; PI3K = phosphatadylinositol 3-kinase; PLC = phospholipase C; SHP2 = tyrosine-protein phosphatase non-receptor type II; SOS = son of sevenless; STAT = signal transducer and activator of transcription; VEGF = vascular endothelial cell growth factor; VEGFR2 = VEGF receptor 2. Modified from Ref. [2].

\section{HUMAN EPIDERMAL GROWTH FACTOR RECEPTOR FAMILY}

The HER family of receptors consists of four members-ErbB-1 (HER1 or epidermal growth factor receptor [EGFR]), ErbB-2 (HER2), ErbB-3 (HER3), and ErbB-4 (HER4) - that share a common structure and regulate cell proliferation, differentiation, and apoptosis when activated to form homo- and heterodimers that facilitate signal transduction [6,7]. All are expressed in gastric cancer, and current attempts to exploit these pathways include

- EGFR-directed therapy

- HER2-directed therapy

- Dual EGFR/HER2-targeted therapy

\subsection{Epidermal Growth Factor Receptor-Directed Therapy}

EGFR is overexpressed in a subset of gastric cancers, making it an attractive target for therapy. Trials to date, however, have failed to demonstrate clinical benefit associated with the addition of an anti-EGFR monoclonal antibody to first-line chemotherapy $[8,9]$. Conducted between June 2008 and December 2010, the phase 3 EXPAND trial randomized 904 patients with metastatic or unresectable, locally advanced gastric cancer to chemo- therapy (cisplatin plus capecitabine) alone or chemotherapy plus cetuximab [8]. Patients were not selected for study based on tumor EGFR expression or KRAS mutation status, nor were patients with HER2-positive disease excluded. Progression-free survival (PFS), a reasonable primary endpoint at the time EXPAND was designed, was not ultimately prolonged in the cetuximab arm (Table 1). Overall survival (OS) and overall response rates (ORR) were similar in the treatment arms, and-unlike in metastatic colorectal cancer-the development of an acne-like rash was not associated with prolonged PFS or OS in the cetuximab arm [8]. Based on these results, cetuximab cannot be recommended for use in combination with first-line chemotherapy for advanced or metastatic gastric cancer.

A possible detrimental effect was reported in REAL3, a randomized trial that evaluated the addition of panitumumab to first-line chemotherapy for locally advanced or metastatic esophagogastric cancer [9]. This open-label phase 3 trial randomized 553 patients with cancer of the esophagus, gastroesophageal junction (GEJ), or stomach to chemotherapy (epirubicin, oxaliplatin, capecitabine [EOC]) alone or chemotherapy plus panitumumab. The trial was designed to detect a $10 \%$ improvement in survival at 1 year; however, it was halted prematurely after a planned interim analysis revealed a statistically inferior OS in the panitumumab group (HR, 1.53; $P=0.0062$ ). At the time of publication, median OS remained significantly shorter in the panitumumab arm (Table 2) [9]. Researchers hope that ongoing biomarker analyses from REAL3 and EXPAND will be informative, but, at the current time, anti-EGFR therapy should not be used in unselected patients outside of a clinical-trial setting.

\subsection{HER2-Directed Therapy}

HER2 is expressed in many tissues, including breast, gastrointestinal tract, kidney, and heart [10]. Up to $30 \%$ of breast cancers overexpress HER2, and these HER2-

Table 1. Results of the randomized phase 3 EXPAND trial.

\begin{tabular}{|c|c|c|c|}
\hline ENDPOINT & $\begin{array}{c}\text { CISPLATIN, } \\
\text { CAPECITABINE, } \\
\text { PLUS } \\
\text { CETUXIMAB }\end{array}$ & $\begin{array}{l}\text { CISPLATINPLUS } \\
\text { CAPECITABINE }\end{array}$ & $\begin{array}{c}\text { HR } \\
(P \text { VALUE })\end{array}$ \\
\hline \multicolumn{4}{|c|}{ Primary endpoints } \\
\hline $\begin{array}{l}\text { Median PFS, } \\
\text { months }\end{array}$ & 4.4 & 5.6 & $\begin{array}{c}1.09 \\
(P=0.32)\end{array}$ \\
\hline \multicolumn{4}{|c|}{ Secondary endpoints } \\
\hline $\begin{array}{l}\text { Median OS, } \\
\text { months }\end{array}$ & 9.4 & 10.7 & $\begin{array}{c}1.00 \\
(P=0.95)\end{array}$ \\
\hline ORR & $30 \%$ & $29 \%$ & NS \\
\hline
\end{tabular}

$\overline{\mathrm{HR}}=$ hazard ratio; NR = not significant; ORR = overall response rate; OS = overall survival; PFS = progression-free survival. Data from Ref. [8]. 
Table 2. Results of the randomized phase 3 REAL3 trial.

\begin{tabular}{cccc}
\hline ENDPOINT & $\begin{array}{c}\text { EOC PLUS } \\
\text { PANITUMUMAB }\end{array}$ & EOC & HR (P VALUE) \\
\hline \multicolumn{4}{c}{ Primary endpoints } \\
$\begin{array}{c}\text { Median OS, } \\
\text { months }\end{array}$ & 8.8 & 11.3 & $1.37(P=0.013)$ \\
$\begin{array}{c}\text { Median PFS, } \\
\text { months } \\
\text { ORR }\end{array}$ & Secondary endpoints & \\
\hline
\end{tabular}

*Odds ratio; EOC = epirubicin, oxaliplatin, capecitabine; HR = hazard ratio; ORR = overall response rate; OS = overall survival; PFS = progression-free survival. Data from Ref. [9].

positive cancers have a poor prognosis and significantly worse clinical outcomes relative to HER2-negative disease. The success of trastuzumab, a monoclonal antibody directed against HER2, and other subsequently developed HER2-targeted therapies, has firmly established HER2-positive breast cancer as a distinct clinical entity and serves as a model for targeting HER2 in other cancers when overexpression or amplification is present [10].

The reported prevalence of HER2 overexpression or amplification in gastric cancer ranges from $8 \%$ to $34 \%$, with higher rates reported for patients with intestinal histology versus diffuse or mixed histology [1,6]. Although the prognostic significance of HER2 in gastric cancer remains controversial, the clinical importance of HER2 cannot be understated. Results from the randomized, phase 3 TOGA study demonstrated that the addition of trastuzumab to cisplatin/fluoropyrimidine therapy extended median survival time by approximately 2 months relative to chemotherapy alone for the first-line treatment of HER2-overexpressing metastatic GEJ adenocarcinoma with no significant differences in the adverse event profile between groups [11]. These data led to the approval of trastuzumab, in combination with cisplatin and capecitabine or 5-fluorouracil (5FU), for this patient population, and such treatment is now considered a standard of care for HER2-positive advanced or metastatic gastric and GEJ adenocarcinoma [1,12].

As in breast cancer, biomarker testing is necessary to identify patients with advanced gastric cancer who may benefit from trastuzumab. There are notable differences in HER2 staining patterns in breast and gastric cancer, however, that necessitate the use of a modified HER2 scoring system for gastric cancer [1]. The modified scoring system has been validated and was used in the pivotal TOGA trial [11]. Briefly, all patients with metastatic disease should now be tested at diagnosis with immunohistochemistry (IHC) or fluorescence in situ hybridization (FISH) as necessary, interpreted as follows [1]:
- An IHC score of 0 or $1+$ is considered negative for HER2 expression

- An IHC score of 2+ is inconclusive and should be confirmed with FISH or another in situ hybridization technique

- An IHC score of 3+ or FISH positive (HER2: CEP17 $\geq 2$ ) is considered HER2 positive

Two additional antibody-based therapies are now available for the treatment of HER2-positive metastatic breast cancer (MBC), and these agents are also being evaluated in gastric cancer (Table 3). Pertuzumab is a monoclonal antibody that prevents the dimerization of HER2 with other HER receptors [10]. It was approved in 2012 for use in combination with trastuzumab and docetaxel for the treatment of patients with HER2-positive MBC who had not received prior anti-HER2 therapy or chemotherapy for metastatic disease based on the results of a randomized placebo-controlled trial that demonstrated significant prolongation of PFS and OS in the pertuzumab treatment arm [13]. In a HER2-overexpressing gastric cancer human xenograft model, the addition of pertuzumab to trastuzumab resulted in enhanced antitumor activity, and the combination is now in clinical development in advanced gastric cancer [7]. A randomized, double-blind, placebo-controlled trial is underway to determine whether the addition of pertuzumab to standard first-line therapy significantly extends OS for patients with HER2-positive metastatic gastric or GEJ cancer. Researchers expect the trial to be completed in 2015 [14].

Ado-trastuzumab emtansine, also known as T-DM1, is an antibody-drug conjugate that combines the tumortargeting properties of trastuzumab with the cytotoxicity of the microtubule inhibitor DM-1 [15]. Upon binding to HER2, T-DM1 is internalized intact; lysosomal degradation leads to the release of the cytotoxic DM1 moiety inside the HER2-positive cell. T-DM1 was approved in 2013 as a single-agent treatment for patients with HER2positive MBC who have previously received trastuzumab and a taxane [16]. In the pivotal randomized controlled trial, T-DM1 significantly prolonged PFS and OS and produced less toxicity than a standard regimen of lapatinib plus capecitabine in this patient population [16]. A andomized phase 3 trial was initiated in the fall of 2012 to examine the safety and efficacy of T-DM1 relative to standard taxane therapy in patients with previously $r$ treated, HER2-positive gastric cancer [17]. OS is the primary endpoint, and the trial is estimated to be completed in 2015 [17]. A smaller phase $1 / 2$ trial is exploring a combination of T-DM1 plus capecitabine in HER2positive MBC and gastric cancer [18].

\subsection{Dual Targeted Therapy}

The oral agent lapatinib, which inhibits HER2 and EGFR, 
Table 3. Clinical trials of pertuzumab-based and ado-TrastuzumabEmtansine therapy for the treatment of HER2-overexpressing advanced or metastatic gastric cancer.

\begin{tabular}{|c|c|c|}
\hline TRIAL IDENTIFIER & DESIGN & STATUS \\
\hline \multicolumn{3}{|c|}{ Fist-Line Pertuzumab Plus Trastuzumab and Chemotherapy Trials } \\
\hline \multirow[b]{2}{*}{ NCT01461057 } & PH2, OL, RCT $(\mathrm{N}=30)$ & \multirow[b]{2}{*}{$\begin{array}{l}\text { Enrollment complete: } \\
\text { awaiting results }\end{array}$} \\
\hline & $\begin{array}{l}\text { Arm 1: Trastuzumab, cisplatin, andcapecitabine plus pertuzumab } 840 \mathrm{mg} \text { loading dose for } \\
\text { cycle } 1 \text { and } 420 \mathrm{mg} \text { for cycle } 2 \text { - 6; pertuzumab is administered every } 3 \text { weeks } \\
\text { Arm 2: Trastuzumab, cisplatin, andcapecitabine plus pertuzumab } 840 \mathrm{mg} \text { for cycle } 1 \text { - 6; } \\
\text { pertuzumab is administered every } 3 \text { weeks }\end{array}$ & \\
\hline \multirow{3}{*}{ NCT01774786 } & PH3, DB, PC, RCT $(\mathrm{N}=780)$ & \multirow{3}{*}{ Recruiting } \\
\hline & $\begin{array}{l}\text { Arm 1: Trastuzumab, cisplatin, andfluropyrimidine (capecitabine or 5FU) plus pertuzumab } \\
\text { ( } 840 \text { mg every } 3 \text { weeks) }\end{array}$ & \\
\hline & $\begin{array}{l}\text { Arm2: Trastuzumab, cisplatin, andfluropyrimidine (capecitabine or 5FU) plus placebo } \\
\text { (every } 3 \text { weeks) }\end{array}$ & \\
\hline \multicolumn{3}{|c|}{ Second-Line Ado-TrastuzumabEmtansine Trials } \\
\hline \multirow{7}{*}{ NCT01641939 } & PH2/3, RCT $(\mathrm{N}=412)$ & \multirow{7}{*}{ Recruiting } \\
\hline & In the first stage, patients will be randomized to : & \\
\hline & Arm1: T-DM1 3.6 mg/kg every 3 weeks & \\
\hline & Arm2: T-DM1 2.4 mg/kg every week & \\
\hline & Arm3: standard taxane therapy ( docetaxel or paxlitaxel per investigator choice ) & \\
\hline & At the end of first stage, the dose and schedule T-DM1 that will be used in second stage & \\
\hline & will be selected & \\
\hline
\end{tabular}

5FU = 5-fluorouracil; DB = double blind; OL = open label; PC = placebo controlled; PH2 = phase 2; PH2/3 = phase 2/3; PH3 = phase 3; RCT = randomized controlled trial; T-DM1 = ado-trastuzumabemtansine. Data compiled from Clinical Trials.gov. http://clinicaltrials.gov. Accessed August 15, 2013.

has been evaluated in the first-line and second-line settings. The phase 3 LOGIC trial randomized 545 patients with HER2-positive advanced or metastatic gastric cancer to chemotherapy (capecitabine and oxaliplatin [CapeOx]) plus lapatinib or chemotherapy plus placebo [19]. Results presented at the American Society of Clinical Oncology (ASCO) annual meeting in June 2013 revealed that the primary endpoint was not achieved; the addition of lapatinib to CapeOx did not significantly improve OS relative to CapeOx plus placebo (median OS, 12.2 vs. 10.5 months; HR, 0.91; $P=0.35$ ), although the results of prespecified subset analyses suggested benefit in Asian patients (HR, 0.68) and those under 60 years of age (HR, 0.69). Toxicity profiles in the study arms were similar, with the exception of increased diarrhea (overall and grade $3+$ ) and overall skin toxicity in the lapatinibtreated patients [19]. Further analysis is needed to better understand the potential role of lapatinib in the first-line setting.

The phase 3 TYTAN trial was conducted in Asia and evaluated the effect of adding lapatinib to second-line treatment with weekly paclitaxel in patients with advanced gastric cancer whose disease progressed after fluoropyrimidine- and/or cisplatin-containing therapy [20]. Patients were required to have HER2 amplification by FISH to be eligible for study enrollment. Interestingly,
HER2 amplification did not routinely correlate to HER2 positivity by IHC in this study, as $35 \%$ of the enrolled patients were found to have IHC 0/1+ scores. In the overall analysis, combination therapy did not significantly prolong OS (11.0 months vs. 8.9 months, respectively; HR, $0.84 ; P=0.21$ ), and the toxicitiy profile was similar to the profile previously reported with lapatinib. In contrast, OS and PFS were significantly longer in the prespecified subgroup of patients with strong HER2 positivity, defined as IHC3+. Median OS was 14.0 months with lapatinib plus paclitaxel versus 7.6 months with paclitaxel alone in this population (HR, $0.59 ; P=0.018$ ). Median PFS was 5.6 months and 4.2 months, respectively (HR, 0.54; $P=0.010$ ) [20]. These results indicate that the definition of HER2 positivity is an important consideration when developing and evaluating HER2targeted therapies [10].

\section{ANGIOGENESIS INHIBITORS}

Angiogenesis, mediated by VEGF through the tyrosine kinase receptors VEGFR-1 and VEGFR-2, is another potential target for the treatment of advanced gastric cancer [21]. VEGF expression is associated with tumor aggressiveness and poor prognosis in gastric cancer, providing a rationale for evaluating angiogenesis inhibitors in this setting [22-24]. 


\subsection{Bevacizumab}

The anti-VEGF monoclonal antibody bevacizumab has demonstrated efficacy in a variety of epithelial carcinomas and was therefore evaluated in the randomized, placebo-controlled, phase 3 AVAGAST trial as a first-line treatment for advanced gastric cancer [21]. From September 2007 to December 2008, 774 patients were randomized to chemotherapy (cisplatin plus a fluoropyrimidine) with bevacizumab or placebo. Median survival, the primary endpoint, was 12.1 months in the bevacizumab arm and 10.1 months in the placebo arm (HR, 0.87; $P=$ 0.10 ), and no new safety signals associated with bevacizumab treatment were identified. Preplanned subgroup analyses revealed that median OS varied widely by geography, with a significant benefit seen in the Americas (HR, 0.63; 95\% CI, 0.43 - 0.94), a trend toward a benefit in Europe (HR, 0.85; 95\% CI, 0.63 - 1.14), but no clear benefit in Asia (HR, 0.97; 95\% CI, 0.75 - 1.25) [21]. Although this finding is intriguing, further investigation is needed to determine whether regional differences in disease presentation, management, or treatment account for the apparent differential efficacy.

Despite failing to meet its primary objective, AVAGAST revealed significant improvements in several secondary endpoints (Table 4). The investigators concluded that bevacizumab has clinical activity in the first-line treatment of advanced gastric cancer, but it is not currently clear how to identify patients for treatment, and it remains investigational for this indication. To this end, AVAGAST contained an extensive predictive biomarker discovery program, and the researchers were able to explore the prognostic and predictive value of various plasma and tissue markers, including [25]:

- Baseline plasma VEGF level

- Tumor VEGF expression

- Tumor VEGFR-2 expression

- Tumor neuropilin-1 expression

Plasma and tumor samples were available from more than $90 \%$ of the AVAGAST patients, and baseline plas- ma VEGF-A level and tumor expression of neuropilin-1, a co-receptor for VEGF, were identified as candidate biomarkers [25]. Patients with high baseline plasma VEGF-A levels appeared to be more sensitive to bevacizumab in terms of OS (HR, 0.72; 95\% CI, $0.57-0.93$ ) than did patients with low levels (HR, 1.01; 95\% CI, 0.77 - 1.31; interaction $P=0.07$ ). Similar results were seen for patients with low baseline expression of neuropilin-1 (HR, 0.75; 95\% CI, 0.59-0.97) versus patients with high neuropilin-1 expression (HR, 1.07; 95\% CI, 0.81 - 1.40; interaction $P=0.06$ ) [25]. It will be critical to validate these biomarkers in prospective clinical trials. Of the two, plasma VEGF-A level may be the most viable candidate for clinical use once the improved, proprietary ELISA used in AVAGAST becomes widely available; however, additional research is needed to identify an appropriate, reproducible cut-off point to distinguish high and low VEGF levels [26]. The manufacturer of bevacizumab has discussed repeating the AVAGAST trial with biomarker-based stratification and is currently recruiting participants for such a trial [26].

\subsection{Ramucirumab}

Ramucirumab, an investigational, fully human monoclonal antibody that targets VEGFR-2, is currently in latestage clinical development for the treatment of gastric cancer, non-small cell lung cancer, hepatocellular carcinoma, colorectal cancer, and breast cancer. In April 2013, its manufacturer received "Fast Track" designation from the FDA to review ramucirumab as a second-line treatment for advanced gastric cancer [27]. Unlike bevacizumab, which targets VEGF directly, ramucirumab targets the extracellular domain of VEGFR-2 [27].

Results from the randomized, placebo-controlled phase 3 REGARD study, one of the largest phase 3 trials in second-line therapy of gastric or GEJ cancer, were presented at the ASCO Gastrointestinal Cancers Symposium in January 2013 and at the $15^{\text {th }}$ World Congress on Gastrointestinal Cancer in July 2013 [28,29]. REGARD

Table 4. Results of the Randomized Phase 3 AVAGAST Trial.

\begin{tabular}{cccc}
\hline ENDPOINT & $\begin{array}{c}\text { CISPLATIN, FLUROPYRIMIDINE, } \\
\text { PLUS BEVACIZUMAB }\end{array}$ & $\begin{array}{c}\text { CISPLATIN, FLUROPYRIMIDINE, } \\
\text { PLUS PLACEBO }\end{array}$ & HR (P VALUE) \\
\hline Median OS, months & 12.1 & Primary endpoints & \\
& & Secondary endpoints & 10.1 \\
Median PFS, months & 6.7 & 5.3 & $0.80(P=0.0037)$ \\
1-year survival rate & $50.2 \%$ & $42.3 \%$ & $P=0.030$ \\
ORR & $46.0 \%$ & $37.4 \%$ & $P=0.032$ \\
\hline
\end{tabular}

$\mathrm{HR}=$ hazard ratio; ORR = overall response rate; OS = overall survival. Data from Ref. [21]. 
was designed to evaluate ramucirumab in patients with metastatic gastric or GEJ adenocarcinoma whose disease progressed after first-line platinum- and/or fluoropyrimidine-containing combination therapy. From October 2009 to January 2012, 355 patients were randomized 2:1 to ramucirumab or placebo, given every 2 weeks until disease progression, unacceptable toxicity, or death. OS, the primary endpoint, was significantly prolonged from 3.8 months in the placebo arm to 5.2 months in the ramucirumab arm (HR, 0.78; $P=0.047)$. Significant improvements were also reported for several secondary endpoints (Table 5). Hypertension occurred more commonly in the ramucirumab group than in the placebo group (16.1\% vs. $7.8 \%$ ); grade 3 hypertension occurred in $7.6 \%$ and $2.6 \%$ of the groups, respectively, but no grade 4 hypertension was observed. Ramucirumab was not associated with higher rates of fatigue, decreased appetite, vomiting, anemia, or other notable toxicities when compared with placebo [28,29]. Both the clinical benefit and rates of grade 3 or higher adverse events associated with ramucirumab treatment appear to compare favorably to the rates associated with second-line chemotherapy reported in other phase 3 trials in this malignancy $[22,30]$.

If approved, ramucirumab could represent an important new treatment option for patients whose disease progresses after first-line treatment. Historically, the role of second-line therapy in metastatic gastric cancer has been unclear, and the most recent guidelines from the National Comprehensive Cancer Network (NCCN), updated in April 2013, list three preferred second-line options-docetaxel, irinotecan, and paclitaxel-based on low-level evidence that these options are appropriate $[1,4,31,32]$. Notably, however, the NCCN has not yet considered the results of the randomized phase 3 COUGAR-2 trial, which compared second-line docetaxel (75 $\mathrm{mg} / \mathrm{m}^{2}$ every 3 weeks for up to 6 cycles) plus active symptom control to active symptom control alone in 168

Table 5. Results of the randomized phase 3 REGARD trial.

\begin{tabular}{cccc}
\hline ENDPOINT & RAMUCIRUMAB & PLACEBO & $\begin{array}{c}\text { HR } \\
(P \text { VALUE })\end{array}$ \\
\hline \multicolumn{4}{c}{ Primary endpoints } \\
$\begin{array}{c}\text { Median OS, } \\
\text { months }\end{array}$ & 5.2 & 3.8 & 0.78 \\
& Secondary endpoints & \\
$\begin{array}{c}\text { Median PFS, } \\
\text { months } \\
\text { ORR }\end{array}$ & 2.1 & 1.3 & $\begin{array}{c}(P<0.047) \\
\text { Disease }\end{array}$ \\
control rate & $3.4 \%$ & $2.6 \%$ & NR \\
\hline
\end{tabular}

$\mathrm{HR}=$ hazard ratio; NR = not reported; ORR = overall response rate; OS = overall survival; PFS = progression-free survival. Data from Refs. [28,29]. patients with locally advanced or metastatic esophagogastric adenocarcinoma that had progressed within 6 months of first-line chemotherapy [31]. As in REGARD, OS, the primary endpoint, was significantly prolonged in the treatment arm, from 3.6 months with active symptom control alone to 5.2 months with docetaxel plus active symptom control (HR, 0.67; $P=0.01$ ) [31]. Docetaxel is not currently indicated for use in the second-line setting, but the results of COUGAR-2 provide high-level evidence of benefit. Together, REGARD and COUGAR-2 demonstrate that fit patients can benefit from additional therapy upon disease progression. The comparative effectiveness of ramucirumab and docetaxel remains unknown, but the results of the ongoing, randomized, placebo-controlled RAINBOW trial, which is comparing second-line ramucirumab plus paclitaxel to paclitaxel plus placebo, will provide additional insight into the role of angiogenesis inhibitors in progressive disease [33].

\section{MET PATHWAY INHIBITORS}

The MET receptor and its ligand, hepatocyte growth factor (HGF), regulate multiple cellular processes associated with cell proliferation, invasion, and angiogenesis. Overexpression of MET occurs in 50\% to $60 \%$ of gastric cancers and is associated with a poor prognosis, making it an attractive candidate for targeted therapy [34,35]. Several inhibitors of the MET/HGF axis are in clinical development, and two-rilotumumab and onartuzumab-are currently in phase 3 trials for the first-line treatment of advanced gastric cancer.

\subsection{Rilotumumab}

Rilotumumab is an investigational, human monoclonal antibody directed against HGF. Activity in advanced gastric cancer was demonstrated in a randomized, double-blind, placebo-controlled phase 2 trial that evaluated the addition of rilotumumab to the combination of epirubicin, cisplatin, and capecitabine (ECX) [36,37]. A total of 121 patients with locally advanced or metastatic gastric or GEJ cancer received ECX in combination with either rilotumumab $15 \mathrm{mg} / \mathrm{kg}$ or $7.5 \mathrm{mg} / \mathrm{kg}$ or placebo. An initial analysis presented in 2011 revealed that median PFS was extended in the combined rilotumumab arm relative to placebo (median PFS, 5.6 vs. 4.2 months; HR, 0.58) [36]. Patients in the rilotumumab-containing arms experienced a higher incidence of peripheral edema, hematologic toxicities, and thromboembolic events than those in the placebo arm [36]. An updated biomarker analysis presented in 2012 demonstrated differential activity by MET protein expression [37]. Briefly, patients with high MET tumor expression benefited from rilotumumab (median OS, 11.1 months) compared with placebo (median OS, 5.7 months; HR 0.29; 95\% CI, 0.11 - 
0.76; $P=0.012$ ) whereas those with low expression had unfavorable survival when treated with rilotumumab (HR, 1.84; 95\% CI, 0.78 - 4.34), suggesting that MET expression may be a predictive marker for rilotumumab treatment benefit [37].

The phase 3, randomized, double-blind, placebo-controlled RILOMET-1 trial was initiated in late 2012 to further evaluate the safety and efficacy of rilotumumab plus ECX as first-line treatment of unresectable, locally advanced or metastatic, HER2-negative, MET-positive gastric or GEJ adenocarcinoma [38]. A total of $450 \mathrm{pa}-$ tients will be enrolled, and the trial should be completed in 2016 [38].

\subsection{Onartuzumab}

Onartuzumab (also known as MetMAb) is a humanized monovalent, or "one-armed", antibody directed against MET. The parent antibody from which onartuzumab was derived has agonist activity and, upon binding MET, induces receptor phosphorylation and downstream signaling; however, the monovalent Fab fragment that comprises onartuzumab is devoid of agonist activity and instead acts as an antagonist [39]. Evidence of activity in gastric cancer was seen in the phase 1 clinical trial, in which one patient with chemotherapy-refractory metastatic gastric cancer obtained a durable complete response to onartuzumab that lasted for 2 years [40].

Onartuzumab is currently being evaluated in the phase 3 , randomized, double-blind, placebo-controlled MetGastric study [41]. A total of 800 patients with HER2-negative, MET-positive, inoperable metastatic gastric or GEJ adenocarcinoma will be randomized to first-line treatment with mFOLFOX6 and either onartuzumab or placebo. Dual primary endpoints are OS in the intentto-treat population and in the MET-positive $(\mathrm{IHC} 2+/ 3+)$ patient subgroup. Enrollment to MetGastric began in late 2012, and the trial should be complete in 2016 [41].

\subsection{Hedgehog Pathway Inhibitors}

The hedgehog signaling pathway, which regulates early embryogenesis and the morphogenesis of specific organs and tissues, is increasingly recognized as having a role in carcinogenesis [42]. In mammals, Hh signaling begins when one of three ligands (Sonic hedgehog, Indian hedgehog, Desert hedgehog) binds to the receptor Patched (PTCH1) [43]. In the absence of ligand binding, PTCH1 works to inhibit the action of the protein Smoothened (SMO), but when binding occurs, PTCH1 releases SMO, which initiates a cascade of events that ultimately leads to the expression of Hh target genes [44]. Activating mutations in the Hh pathway are responsible for some sporadic and familial basal cell carcinomas (BCC) and medulloblastomas, whereas overexpression is the prima- ry aberration in other tumor types [43].

Vismodegib, an oral small-molecule $\mathrm{Hh}$ antagonist that inhibits the Hh pathway by binding SMO and interfering with the growth and survival of cells, is indicated for the treatment of adults with metastatic BCC as well as those who have locally advanced BCC that has recurred following surgery or who are not candidates for surgery, and who are not candidates for radiation [45]. It is under active investigation in other tumor types, including gastric cancer. Although Hh signaling has a variety of effects in the stomach, dysregulated ligand expression appears to have a role in gastric carcinogenesis, and overexpression occurs in gastroesophageal tumors [46,47]. Preliminary results presented at ASCO in 2013 of a randomized phase 2 trial examining the effects of vismodegib in 124 patients with untreated metastatic or locally advanced gastric or GEJ adenocarcinoma suggested no improvement in the primary endpoint of PFS [47]. However, researchers are conducting blood and tissue biomarker analyses to determine whether a subset of patients may benefit from this treatment approach [47].

\section{CONCLUSION}

Molecular-targeted therapy is changing the treatment of gastric cancer. The addition of trastuzumab to first-line chemotherapy is now a standard of care for the treatment of HER2-positive advanced or metastatic disease, and other HER2-targeted therapies are in late-stage clinical development. Most patients, however, do not have HER2-positive disease, and other options are needed. To date, EGFR-targeted therapies have not been proved effective in unselected patients, but ongoing biomarker analyses of phase 3 trials may ultimately identify a subgroup that can benefit from these agents. Work is also underway to identify biomarkers that predict benefit from the antiangiogenesis agent bevacizumab in advanced gastric cancer. In contrast to first-line bevacizumab-based therapy, the investigational angiogenesis inhibitor ramucirumab has been shown to significantly extend survival in the second-line setting and is currently under FDA review. The MET pathway inhibitors, rilotumumab and onartuzumab, are currently being evaluated in randomized phase 3 trials, with results expected in several years. It is hoped that these and other advances will improve the prognosis and extend survival for patients with advanced and metastatic gastric and GEJ adenocarcinoma.

\section{ACKNOWLEDGEMENTS}

This research was supported by the grants from Shanghai Commission for Science and Technology (KSCX2-YW-R-112), and Shanghai Leading Academic Discipline Project (J50101). 


\section{CONFLICT OF INTEREST AND AUTHORS' CONTRIBUTION}

- The manuscript has not been published previously and is not under consideration (in whole or in part) for publication elsewhere.

- All authors contributed in conception and design; analysis and interpretation of the data; drafting of the article; critical revision of the article for important intellectual content; final approval of the article

\section{CONFLICT OF INTEREST}

We declare that we do not have any source of funding mentioned and there is no conflicts of interest disclosed. We disclose no financial and personal relationship with other people or organizations that could inappropriately influence their work.

\section{REFERENCES}

[1] National Comprehensive Cancer Network (2013) NCCN clinical practice guidelines in oncology (NCCN Guidelines $\left.{ }^{\circledR}\right)$ : Gastric cancer, version 2. www.nccn.org

[2] Smyth, E.C. and Cunningham, D. (2012) Targeted therapy for gastric cancer. Current Treatment Options in Oncology, 13, 377-389.

http://dx.doi.org/10.1007/s11864-012-0192-6

[3] Guggenheim, D.E. and Shah, M.A. (2013) Gastric cancer epidemiology and risk factors. Journal of Surgical Oncology, 107, 230-236.

http://dx.doi.org/10.1002/jso.23262

[4] Price, T.J., Shapiro, J.D., Segelov, E., et al. (2012) Management of advanced gastric cancer. Expert Review of Gastroenterology \& Hepatology, 6, 199-208; quiz 209. http://dx.doi.org/10.1586/egh.11.103

[5] DeVita, F., Giuliani, F., Silvestris, N., et al. (2012) Current status of targeted therapies in advanced gastric cancer. Expert Opinion on Therapeutic Targets, 16, S29-S34. http://dx.doi.org/10.1517/14728222.2011.652616

[6] Begnami, M.D., Fukuda, E., Fregnani, J.H., et al. (2011) Prognostic implications of altered human epidermal growth factor receptors (HERs) in gastric carcinomas: HER2 and HER3 are predictors of poor outcome. Journal of Clinical Oncology, 29, 3030-3036. http://dx.doi.org/10.1200/JCO.2010.33.6313

[7] Yamashita-Kashima, Y., Iijima, S., Yorozu, K., et al. (2011) Pertuzumab in combination with trastuzumab shows significantly enhanced antitumor activity in HER2-positive human gastric cancer xenograft models. Clinical Cancer Research, 17, 5060-5070. http://dx.doi.org/10.1158/1078-0432.CCR-10-2927

[8] Lordick, F., Kang, Y.K., Chung, H.C., et al. (2013) Arbeitsgemeinschaft Internistische Onkologie and EXPAND Investigators. Capecitabine and cisplatin with or without cetuximab for patients with previously untreated advanced gastric cancer (EXPAND): A randomised, open-label phase 3 trial. Lancet Oncology, 14, 490-499. http://dx.doi.org/10.1016/S1470-2045(13)70102-5

[9] Waddell, T., Chau, I., Cunningham, D., et al. (2013) Ep- irubicin, oxaliplatin, and capecitabine with or without panitumumab for patients with previously untreated advanced oesophagogastric cancer (REAL3): A randomised, open-label phase 3 trial. Lancet Oncology, 14, 481-489. http://dx.doi.org/10.1016/S1470-2045(13)70096-2

[10] Boku, N. (2013) HER2-positive gastric cancer. Gastric Cancer, 17, 1-12.

[11] Bang, Y.-J., Van Cutsem, E., Feyereislova, A., et al. for ToGA Trial Investigators (2010) Trastuzumab in combination with chemotherapy versus chemotherapy alone for treatment of HER2-positive advanced gastric or gastrooesophageal junction cancer (ToGA): A phase 3, openlabel, randomised controlled trial. Lancet, 376, 687-697. http://dx.doi.org/10.1016/S0140-6736(10)61121-X

[12] Herceptin [package insert]. (2010) South San Francisco, CA: Genentech, Inc.

[13] Perjeta [package insert]. (2012) South San Francisco, CA: Genentech, Inc.

[14] Clinical Trials.gov. (2013) A study of Perjeta (pertuzumab) in combination with Herceptin (trastuzumab) and chemotherapy in patients with HER2-positive metastatic gastroesophageal junction or gastric cancer. http://clinicaltrials.gov/ct2/show/record/NCT01774786

[15] Verma, S., Miles, D., Gianni, L., et al. for the EMILIA Study Group (2012) Trastuzumab emtansine for HER2positive advanced breast cancer. New England Journal of Medicine, 367, 1783-1791. http://dx.doi.org/10.1056/NEJMoa1209124

[16] Kadcyla [package insert]. (2013) South San Francisco, CA: Genentech, Inc.

[17] Clinical Trials.gov. (2013) A study of trastuzumab emtansine versus taxane in patients with advanced gastric cancer. http://clinicaltrials.gov/ct2/show/NCT01641939

[18] Clinical Trials.gov. (2013) A combination study of trastuzumab emtansine and capecitabine in patients with breast cancer or gastric cancer. http://clinicaltrials.gov/ct2/show/NCT01702558

[19] Hecht, J.R., Bang, Y.-J., Qin, S., et al. (2013) Lapatinib in combination with capecitabine plus oxaliplatin (CapeOx) in HER2-positive advanced or metastatic gastric, esophageal, or gastroesophageal adenocarcinoma (AC): The TRIO-013/LOGiC Trial. Journal of Clinical Oncology, 31, abstr LBA4001.

[20] Bang, Y.-J., Ruihua, X., Taroh, S., et al. (2013) A randomized, open-label, phase III study of lapatinib in combination with weekly paclitaxel versus weekly paclitaxel alone in the second-line treatment of HER2 amplified advanced gastric cancer in Asian population: TyTAN study. 2013 Gastrointestinal Cancers Symposium, San Francisco, 24-26 January 2013.

[21] Ohtsu, A., Shah, M.A., Van Cutsem, E., et al. (2011) Bevacizumab in combination with chemotherapy as first-line therapy in advanced gastric cancer: A randomized, double-blind, placebo-controlled phase III study. Journal of Clinical Oncology, 29, 3968-3976. http://dx.doi.org/10.1200/JCO.2011.36.2236

[22] Kang, J.H., Lee, S.I., Lim do, H., et al. (2012) Salvage chemotherapy for pretreated gastric cancer: A randomized 
phase III trial comparing chemotherapy plus best supportive care with best supportive care alone. Journal of Clinical Oncology, 30, 1513-1518. http://dx.doi.org/10.1200/JCO.2011.39.4585

[23] Kim, S.E., Shim, K.N., Jung, S.A., Yoo, K. and Lee, J.H. (2009) The clinicopathological significance of tissue levels of hypoxia-inducible factor-1alpha and vascular endothelial growth factor in gastric cancer. Gut and Liver, 3, 88-94. http://dx.doi.org/10.5009/gnl.2009.3.2.88

[24] Maeda, K., Chung, Y.S., Ogawa, Y., et al. (1996) Prognostic value of vascular endothelial growth factor expression in gastric carcinoma. Cancer, 77, 858-863. http://dx.doi.org/10.1002/(SICI)1097-0142(19960301)77: 5<858::AID-CNCR8>3.0.CO;2-A

[25] Van Cutsem, E., De Haas, S., Kang, Y.K., et al. (2012) Bevacizumab in combination with chemotherapy as first-line therapy in advanced gastric cancer: A biomarker evaluation from the AVAGAST randomized phase III trial. Journal of Clinical Oncology, 30, 2119-2127. http://dx.doi.org/10.1200/JCO.2011.39.9824

[26] Maru, D., Venook, A.P. and Ellis, L.M. (2013) Predictive biomarkers for bevacizumab: Are we there yet? Clinical Cancer Research, 19, 2824-2827. http://dx.doi.org/10.1158/1078-0432.CCR-12-3409

[27] Reichert, J.M. (2013) Antibodies to watch in 2013: Midyear update. MAbs, 5, 513-517.

http://dx.doi.org/10.4161/mabs.24990

[28] Fuchs, C.S., Tomasek, J., Cho, J.Y., et al. (2013) REGARD: A phase III, randomized, double-blinded trial of ramucirumab and best supportive care (BSC) versus placebo and BSC in the treatment of metastatic gastric or gastroesophageal junction (GEJ) adenocarcinoma following disease progression on first-line platinum- and/or fluoropyrimidine-containing combination therapy. Journal of Clinical Oncology, 31, abstr LBA5.

[29] Tabernero, J., Tomasek, J., Filip, D., et al. (2013) REGARD phase 3, randomized trial of ramucirumab in patients with metastatic gastric or GEJ adenocarcinoma following progression on first-line chemotherapy. Annals of Oncology, 24, iv14.

http://dx.doi.org/10.1093/annonc/mdt201.8

[30] Ford, H., Marshall, A., Wadsley, J., et al. (2013) Cougar-02: A randomized phase III study of docetaxel versus active symptom control in advanced esophagogastric adenocarcinoma. Journal of Clinical Oncology, 30, abstr LBA4.

[31] Wesolowski, R., Lee, C. and Kim, R. (2009) Is there a role for second-line chemotherapy in advanced gastric cancer? The Lancet Oncology, 10, 903-912. http://dx.doi.org/10.1016/S1470-2045(09)70136-6

[32] Cervantes, A., Roda, D., Tarazona, N., Roselló, S. and Pérez-Fidalgo, J.A. (2013) Current questions for the treatment of advanced gastric cancer. Cancer Treatment Reviews, 39, 60-67. http://dx.doi.org/10.1016/j.ctrv.2012.09.007

[33] Clinical Trials.gov. (2013) A study of paclitaxel with or without ramucirumab in metastatic gastric adenocarcinoma (RAINBOW).

http://clinicaltrials.gov/ct2/show/NCT01170663
[34] Graziano, F., Galluccio, N., Lorenzini, P., et al. (2011) Genetic activation of the MET pathway and prognosis of patients with high-risk, radically resected gastric cancer. Journal of Clinical Oncology, 29, 4789-4795. http://dx.doi.org/10.1200/JCO.2011.36.7706

[35] Huang, T.J., Wang, J.Y., Lin, S.R., Lian, S.T. and Hsieh, J.S. (2001) Overexpression of the c-met protooncogene in human gastric carcinoma-Correlation to clinical features. Acta Oncologica, 40, 638-643. http://dx.doi.org/10.1080/028418601750444204

[36] Iverson, T., Donehower, R.C., Davidenko, I., et al. (2011) Safety and efficacy of epirubicin, cisplatin and capecitabine (ECX) plus rilotumumab (R) as first-line treatment for unresectable locally advanced (LA) or metastatic (M) gastric or esophagogastric junction (EGJ) adenocarcinoma. European Journal of Cancer, 47, S443. http://dx.doi.org/10.1016/S0959-8049(11)71815-9

[37] Oliner, K.S., Tang, R., Anderson, A., et al. (2012) Evaluation of MET pathway biomarkers in a phase II study of rilotumumab (R, AMG 102) or placebo (P) in combination with epirubicin, cisplatin and capecitabine (ECX) in patients (pts) with locally advanced or metastatic gastric (G) or esophagogastric junction (EGJ) cancer. Journal of Clinical Oncology, 30, abstr 4005.

[38] Clinical Trials.gov. (2013) First-line treatment for locally advanced or metastatic mesenchymal epithelial transition factor (MET)-positive gastric, lower esophageal, or gastroesophageal junction (GEJ) adenocarcinoma (RILOMET-1). http://clinicaltrials.gov/show/NCT01697072

[39] Surati, M., Patel, P., Peterson, A. and Salgia, R. (2011) Role of MetMAb (OA-5D5) in c-MET active lung malignancies. Expert Opinion on Biological Therapy, 11, 1655-1662. http://dx.doi.org/10.1517/14712598.2011.626762

[40] Catenacci, D.V.T., Henderson, L., Xiao, S.-Y., et al. (2011) Durable complete response of metastatic gastric cancer with anti-met therapy followed by resistance at recurrence. Cancer Discovery, 1, 573-579. http://dx.doi.org/10.1158/2159-8290.CD-11-0175

[41] Clinical Trials.gov. (2013) A study of onartuzumab (MetMAb) in combination with mFOLFOX6 in patients with metastatic HER2-negative and MET-positive gastroesophageal cancer (MetGastric). http://clinicaltrials.gov/ct2/show/NCT01662869

[42] McMillan, R. and Matsui, W. (2012) Molecular pathways: The hedgehog signaling pathway in cancer. Clinical Cancer Research, 18, 4883-4888. http://dx.doi.org/10.1158/1078-0432.CCR-11-2509

[43] Ng, J.M.Y. and Curran, T. (2011) The hedgehog's tale: Developing strategies for targeting cancer. Nature Reviews Cancer, 11, 493-501. http://dx.doi.org/10.1038/nrc3079

[44] Hui, C. and Angers, S. (2011) Gli proteins in development and disease. Annual Review of Cell and Developmental Biology, 27, 513-537.

http://dx.doi.org/10.1146/annurev-cellbio-092910-154048

[45] Erivedge [package insert]. (2012) South San Francisco, CA: Genentech, Inc. 
[46] Martin, J., Donnelly, J.M., Houghton, J.M. and Zavros, Y. (2010) The role of sonic hedgehog reemergence during gastric cancer. Digestive Diseases and Sciences, 55, 1516-1524. http://dx.doi.org/10.1007/s10620-010-1252-z

[47] Cohen, D.J., Christos, P.J., Kindler, H.L., et al. (2013) Vismodegib (V), a hedgehog pathway inhibitor, combined with FOLFOX for the first-line therapy of patients (pts) with advanced gastric cancer and gastroesophageal junction (GEJ) carcinoma: A New York Cancer Consortium led phase II randomized study. Journal of Clinical Oncology, 21, abstr 4011. 